\title{
Atomic-Level-Designed Catalytically Active Palladium Atoms on Ultrathin Gold Nanowires
}

\author{
Rui Liu, Li-Qiang Zhang, Cun Yu, Meng-Tao Sun, Jing-Fu Liu,* and Gui-Bin Jiang
}

Catalytically active sites of heterogeneous catalysts generally are surface-bound atoms that are accessible to reactants. ${ }^{[1]}$ The atomic efficiencies (approximately the percentage of surface atoms) and per metal atom-based activity of active sites therefore determine the overall catalytic performance of a nanocatalyst. ${ }^{[2]}$ Atomic efficiency can be maximized using the well-developed monolayer $(\mathrm{ML})^{[3]}$ or even single-atom strategy. ${ }^{[2,4]}$ While the atomic geometry of the active site is a central influencer on catalytic activity, in some cases a single atom can serve as an active site. ${ }^{[4 a, b]}$ For example, individual palladium atoms activate $\mathrm{H}_{2}$ in a heterolytic pathway and show 9 and 55 times higher activity over commercial Pd catalysts during the hydrogenation of $\mathrm{C}=\mathrm{C}$ bonds/aldehydes, respectively. ${ }^{[2]}$ By contrast, reactions like the acetylation of ethylene and the electrooxidation of formic acid/methanol require at least two contiguous atoms..$^{[5]}$ Moreover, optimizing the atom arrangement in the active site also gives high selectivity for a desired product. ${ }^{[5,6]}$ Therefore, the ability to directly manipulate the geometry of the catalytically active atoms (e.g., continuous vs isolated) is highly desired. ${ }^{[7]}$ While great strides have been made in constructing single $e^{[4 \mathrm{~b}, \mathrm{c}, 8]}$ and continuous atoms (on large surface area supports and metal nanocrystals, respectively ${ }^{[6 a, 9]}$ for use as efficient catalysts, the ability to conveniently switch the catalytically active atoms between isolateddominated and ensemble-dominated on similar supports is still in its infancy. ${ }^{[4 \mathrm{~b}, 10]}$ To this end, we are particularly interested in the atomic geometry of Pd atoms on Au surface. Pd plays an indispensable role in a number of reactions, and the incorporation of $\mathrm{Au}$ atoms to form AuPd bimetal nanocatalyst result in largely increased activity and longevity. ${ }^{[5 a, 9 a, 11]}$ Design the next generation of AuPd catalysts requires a better understanding of how the geometry, ligand, and electron effect influences the

\footnotetext{
Dr. R. Liu, Prof. J.-F. Liu, Prof. G.-B. Jiang

State Key Laboratory of

Environmental Chemistry and Ecotoxicology

Research Center for Eco-Environmental Sciences

Chinese Academy of Sciences

Beijing 100085, China

E-mail: jfliu@rcees.ac.cn

Dr. L.-Q. Zhang, Dr. C. Yu

State Key Laboratory of Heavy Oil Processing and

Department of Materials Science and Engineering

China University of Petroleum

Beijing 102249, China

Prof. M.-T. Sun

Beijing National Laboratory for Condensed Matter Physics

Institute of Physics

Chinese Academy of Sciences

Beijing 100190, China
}

DOI: 10.1002/adma.201604571 activity of the catalysts. ${ }^{[5 a, b, 7,12]}$ This depends on the preparation of geometry-specific Pd atoms on Au or likely substrate. However, unlike the convenient switch between isolated/contiguous $\mathrm{Pd}$ atoms on ideal single-crystal $\mathrm{Au}$ surface, ensemble rather than isolated $\mathrm{Pd}$ forms on $\mathrm{Au}$ nanostructures, even the coverage of $\mathrm{Pd}$ is as low as $0.05 \mathrm{ML} \cdot{ }^{[12,13]}$ This is partially for the weak interaction between the $\mathrm{Pd}$ and $\mathrm{Au}$ and makes the provision of atomic geometry specific AuPd nanocatalysts very challenging.

Herein, we propose a new conceptual strategy for manipulating the growth pattern of shell $\mathrm{Pd}$ atoms, i.e., by finely tuning the interactions between the core and shell metal atoms. The feasibility of this method was shown through our success in tailoring the atomic geometry of ML Pd atoms on ultrathin $\mathrm{Au}$ nanowires (NWs), where an Ag ML was predeposited on $\mathrm{Au}\left(\mathrm{Au} @ \mathrm{Ag}_{\mathrm{ML}}\right.$ ) and changes the core and the shell metals from $\mathrm{Au}-\mathrm{Pd}$ to $\mathrm{Au} / \mathrm{Ag}-\mathrm{Pd}$. Au@Ag $\mathrm{Ag}_{\mathrm{ML}}$ efficiently stabilizes the deposited $\mathrm{Pd}$ atoms and facilitates the formation of isolated atoms on $\mathrm{Au} @ \mathrm{Ag}_{\mathrm{ML}}$ NWs rather than contiguous Pd atoms on $\mathrm{Au}$ NWs. The strengthened $\mathrm{Au} / \mathrm{Ag}-\mathrm{Pd}$ interaction is confirmed by measuring the surface-enhanced Raman spectra (SERS) of chemisorbed 2,6-dimethylphenylisocyanide (2,6-DMPI) and $\mathrm{X}$-ray adsorption spectroscopy (XAS). In addition, the relative abundances of isolated and contiguous Pd atoms were estimated by SERS spectra. This is correlated by testing the catalytic activity of the $\mathrm{Pd}$ atoms in the hydrogenation of 4-nitrophenol (4-NP) and the electrooxidation of ethanol. Impressively, the atomic geometry plays a central role in determining the catalytic activity of the Pd atoms, which even exceeded the influence from the core metal contents (i.e., the $\mathrm{Au}$ or the $\mathrm{Au} / \mathrm{Ag}$ atoms). ${ }^{[4 \mathrm{~b}, 10,14]}$ The isolated and the continuous $\mathrm{Pd}$ atoms are identified as the active sites for the reduction of 4-NP and the electrocatalyzed oxidation of ethanol, respectively. To the best of our knowledge, this is the first report on the manipulating the Pd atomic geometry at the ML-level, and therefore the direct correlation of catalytic activity for active sites possessing a defined geometry at the atomic scale.

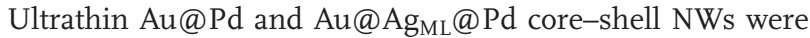
synthesized using a recently reported method. ${ }^{[5 c]}$ Their atomic structures and 1D morphology as visualized by low-magnification transmission/scanning electron microscope are presented in Figure 1a and Figures S1 and S2 (Supporting Information), respectively. The presence of $\mathrm{Ag}$ and $\mathrm{Pd}$ atoms on the surface of the NWs was confirmed using energy-dispersive X-ray spectroscopy (EDS) elemental mapping (Figure 1b-d). Here, Au atoms are concentrated in the core of the NWs, while Ag and Pd are distributed homogeneously on the surface of the NWs. In order to distinguish the $\mathrm{Pd} / \mathrm{Ag}$ shell from the $\mathrm{Au}$ core, spherical aberration-corrected high-angle-annular-dark-field scanning transmission electron microscopy (Cs-HAADF-STEM) was used to characterize Au@Ag $\mathrm{Ag}_{\mathrm{ML}}$, Au@Pd, and Au@Ag $\mathrm{AL} @ P d$ 

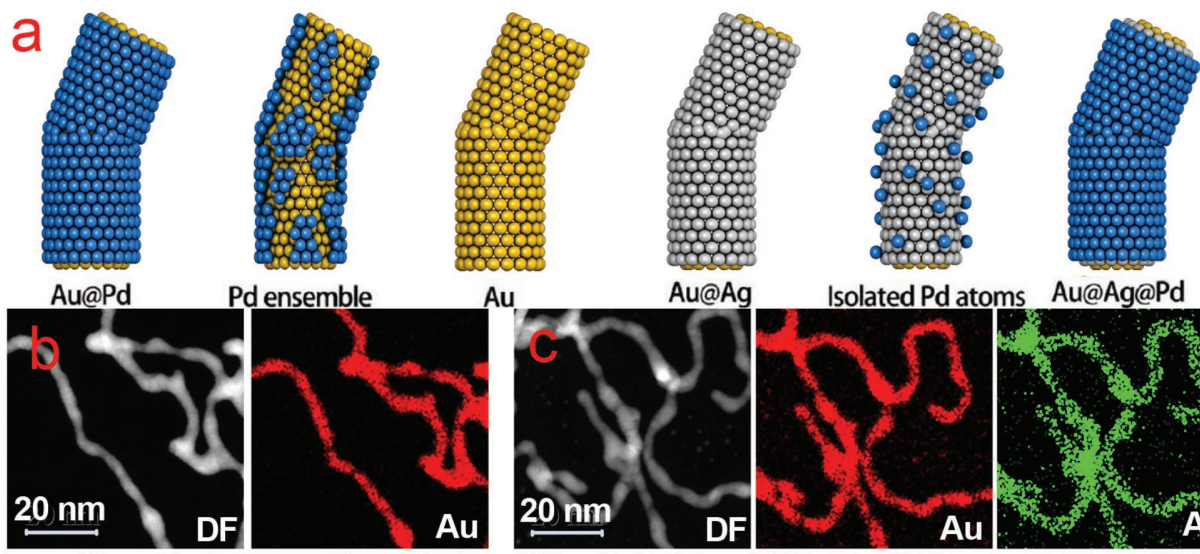

Isolated Pd atoms Au@Ag@Pd
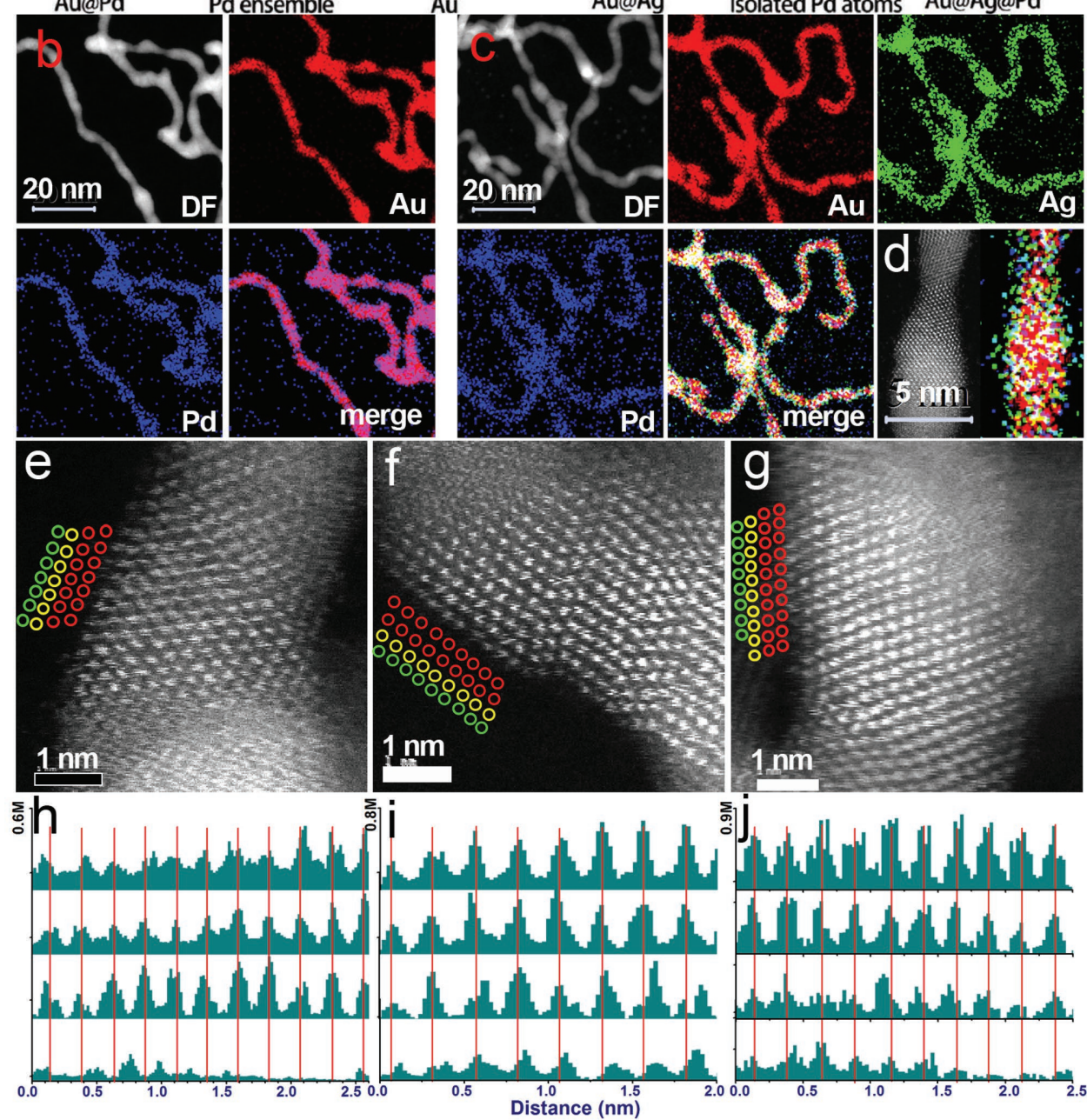

Figure 1. a) Schematic illustration of tailoring the atomic geometry of Pd atoms on the Au NW surface using a Ag ML. b,c) HAADF-STEM images and the corresponding STEM-EDS elemental mappings of Au@Pd and Au@Ag ${ }_{M L} @ P d$ NWs (scale bar, 20 nm).d) High-resolution HAADF-STEM images and corresponding STEM-EDS elemental mappings of Au@Ag $@$ MLPd NW (scale bar, $5 \mathrm{~nm}$ ). e-g) Atomic resolution Cs-HAADF-STEM images of $A u @ A g, A u @ P d$, and $A u @ A g$ ML $@ P d N W s$, and h-j) the corresponding intensity profile of the outer four atomic layers.

NWs (Figure 1e-j). For areas where atomic resolution was achieved, one (for $\mathrm{Au@Ag}$ and $\mathrm{Au@Pd)} \mathrm{or} \mathrm{two} \mathrm{atomic} \mathrm{layers}$ (for Au@Ag $\mathrm{AL}_{\mathrm{ML}} @ \mathrm{Pd}$ ) were observed to have significantly different atomic arrangements. Moreover, STEM intensity analysis revealed a brightness contrast between the core and shell atoms due to differences in atomic numbers (Z). EDS line scanning showed that the intensity of Pd/Ag was slightly higher in the outer shell than the inner area (Figure S3, Supporting Information). ${ }^{[15]}$ Together, these results support our conclusion that an $\mathrm{ML}$ of $\mathrm{Ag} / \mathrm{Pd}$ is epitaxially grown on $\mathrm{Au} \mathrm{NWs.}{ }^{[16]}$ In agreement with the Cs-HAADF-STEM observation, the surface plasmon resonance properties of the NWs also suggest that there was a Pd/Ag layer on the Au surface (Figure S4, Supporting Information). Furthermore, synchrotron X-ray diffraction (Syn-XRD) and elemental analysis indicate that there was no large cluster of either single-phase $\mathrm{Pd}$ or $\mathrm{Ag}$, and the majority of the $\mathrm{Ag}$ and Pd precursors were coated onto the Au NWs (Figures S5 and S7, Supporting Information).

Next, we investigated the interaction between $\mathrm{Au} / \mathrm{Ag}$ and Pd. This was done by measuring the SERS of chemisorbed 2,6-DMPI, which is isoelectronic with $\mathrm{CO}$ but has a large Raman cross section, and is widely employed in interrogating the electronic structure of transition metal atoms. ${ }^{[17]}$ Once 2,6-DMPI had chemisorbed onto the Au@Ag NWs, there was 


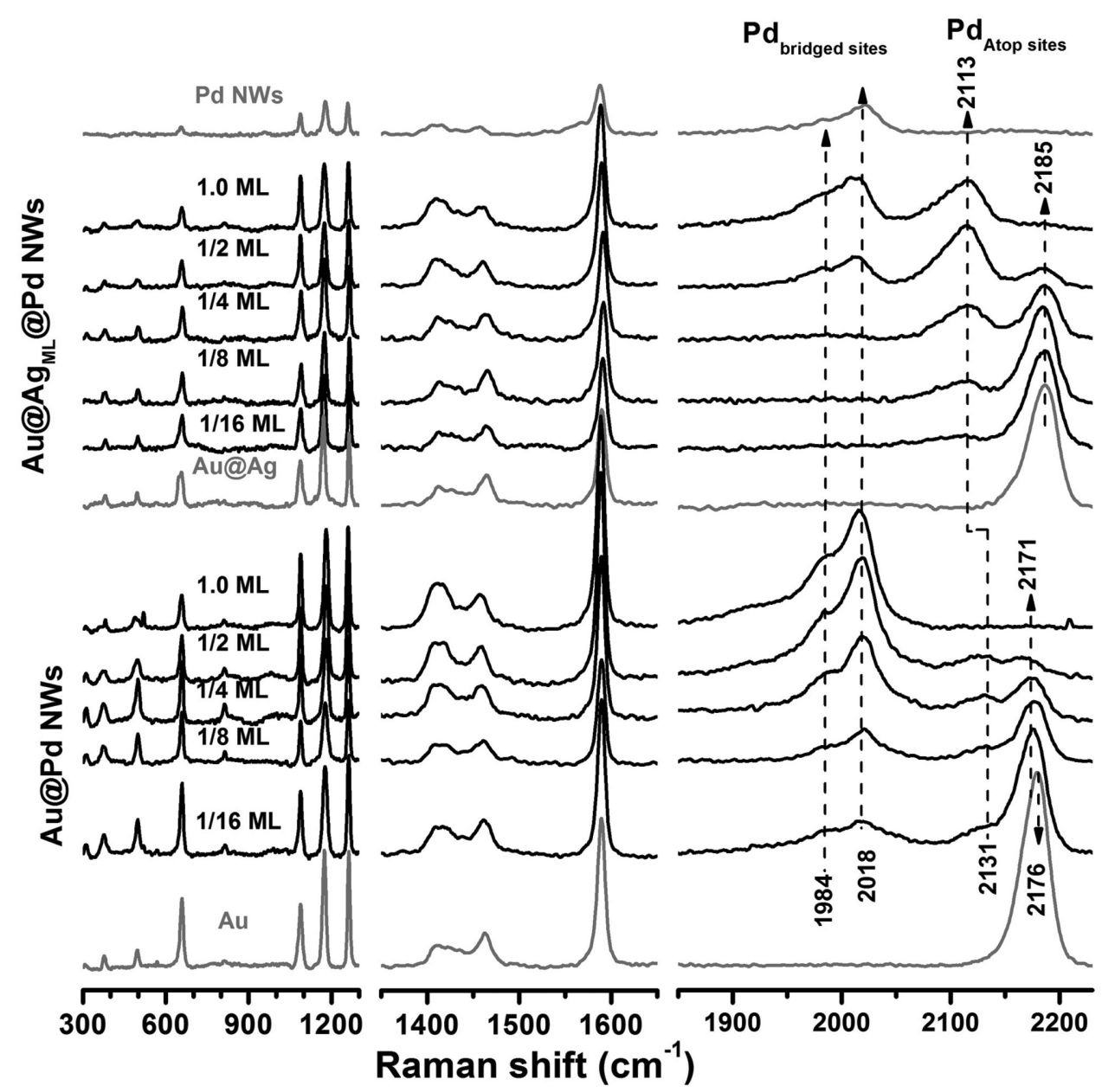

Figure 2. SERS spectra of 2,6-DMPI chemisorbed on Au, Au@Pd, Au@Ag, Au@Ag $g_{M L} @ P d$, and Pd NWs. From bottom to top, the Pd coverage is $1 / 16,1 / 8,1 / 4,1 / 2$, and $1.0 \mathrm{ML}$, respectively.

a blue-shifted in the $v_{\mathrm{NC}}$ stretch mode, from $\approx 2167$ (Ag nanostructure) to $2185 \mathrm{~cm}^{-1}$ (Figure 2; Figures S8 and S9, Supporting Information). In addition, there was a blue-shift of $9 \mathrm{~cm}^{-1}$ from $\mathrm{Au}\left(2176 \mathrm{~cm}^{-1}\right)$, indicating enhanced $\delta$-donation from the $\mathrm{C} \equiv \mathrm{N}$ bond to the $d$-band of the Ag atoms on the Au@ Ag NWs. Since Ag has a lower electron affinity than $A u,{ }^{[17 a]}$ this blue-shifted Raman frequency was likely due to the lower $d$-electron density of the $\mathrm{Ag}$ atoms, or $\mathrm{Ag}$ transfer $d$-electrons to $\mathrm{Au}$. In addition, the $v_{\mathrm{NC}}$ band of the 2,6-DMPI atop bounded on Pd sites in the Au@Ag $\mathrm{Ag}_{\mathrm{ML}} @ \mathrm{Pd}$ NWs shows an $18 \mathrm{~cm}^{-1}$ red shift relative to the Au@Pd NWs. This suggests the enhanced $\pi$-back donation from the $d$-band of the Pd atom to the $\pi^{*}$ orbital of 2,6-DMPI, or Pd atoms in the Au@Ag @L @Pd NWs are in a higher $d$-electron filling state over their counterparts in the Au@Pd NWs. This is ascribed to an electron withdrawal/ charge transfer effect from the $\mathrm{Au} / \mathrm{Ag}$ atoms to $\mathrm{Pd}$ atoms, which was further verified by X-ray photoelectron spectroscopy (Figure S10, Supporting Information).

The SERS spectra of chemisorbed 2,6-DMPI also provide structural information of the shell Pd atoms. Similar to 2,6DMPI adsorbed on Pd NWs, the $v_{\mathrm{NC}}$ band of the 2,6-DMPI anchored on Pd in the Au@Pd NWs is primarily observed at $\approx 2000 \mathrm{~cm}^{-1}$. This is consistent with 2,6-DMPI adsorbed on bridge sites of continuous $\mathrm{Pd}$ atoms ${ }^{[5 \mathrm{~b}]}$ and suggests that the deposited Pd atoms drifted on the Au NW surface and formed Pd ensembles. However, this band was only observed on the Au@Ag $\mathrm{AL}_{\mathrm{ML}} @ \mathrm{Pd}$ NWs when the Pd coverage was 1/4 ML or above, while the dominant peak was located at $\approx 2100 \mathrm{~cm}^{-1}$, indicative of 2,6-DMPI bound to Pd atop sites (see Discussion and Figure S9, Supporting Information), in line with previous publications. ${ }^{[5 a, b]}$ Since the atop binding is energetically less favorable than bridged/hollow adsorption, ${ }^{[17 \mathrm{~b}]}$ the atop-chemisorption in Au@Ag@Pd NWs renders the unavailability of alternative atomic sites. These spectroscopic differences suggest the presence of an $\mathrm{Ag}$ ML tailors the atomic geometry of the Pd atoms. In the Au@Pd NWs, Pd likely exists as continuous atoms in contrast to $\mathrm{Au} @ \mathrm{Ag}_{\mathrm{ML}} @ \mathrm{Pd}$ NWs, where the formation of isolated Pd atoms is favored.

The above SERS result, namely, the strengthened interaction between deposited Pd atoms and $\mathrm{Au@Ag}$ ML NWs and the changed Pd atomic geometry, is further verified by XAS. ${ }^{[18]}$ In the Pd k-edge X-ray absorption near-edge structure spectra, where the white line intensity decreases in the order of $\mathrm{Pd} \mathrm{NWs}>\mathrm{Pd}$ foil > Au@Pd NWs > Au@Ag $\mathrm{AL}_{\mathrm{ML}} @ \mathrm{Pd}$ NWs (Figure S11, Supporting Information). This, in one hand, shows the enhanced antioxidation capacity of $\mathrm{Pd}$ atoms in close contact with $\mathrm{Au}$ or 

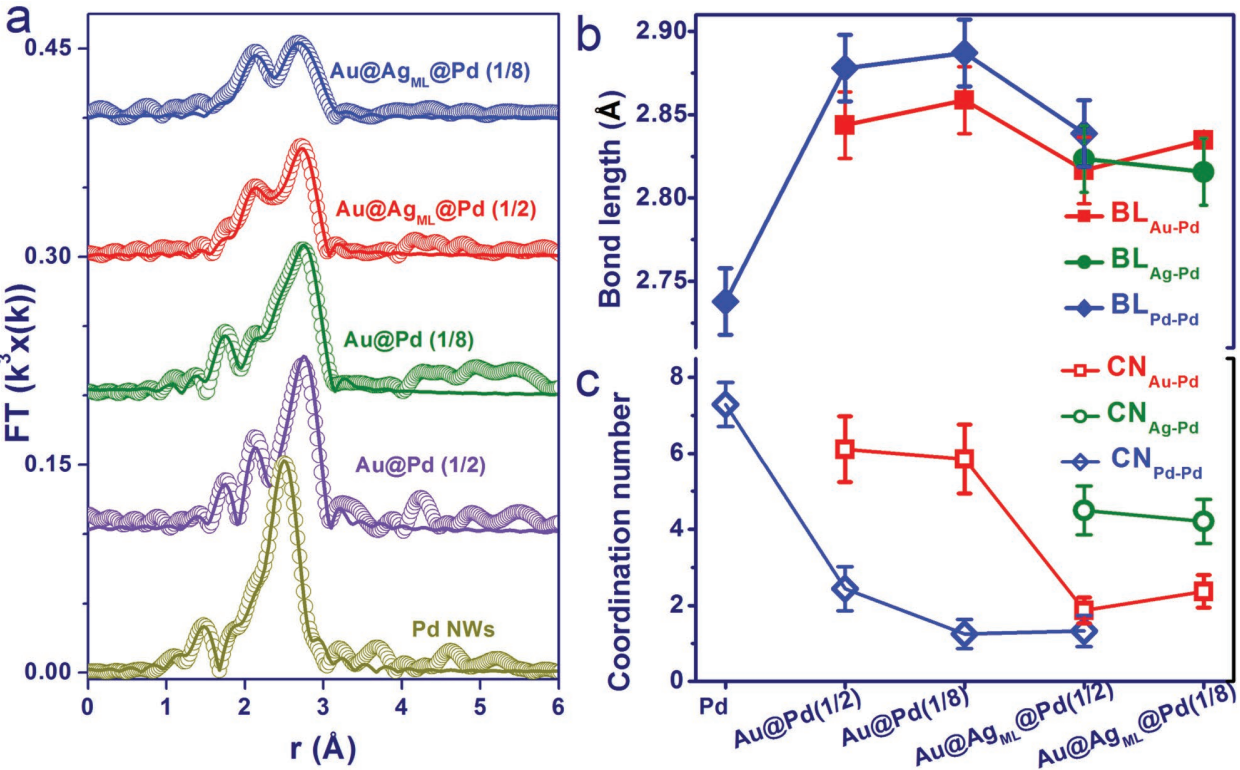

Figure 3. a) The Fourier transformed $k^{3}$-weighted Pd k-edge EXAFS spectra, b,c) and experimentally determined $\mathrm{Au} / \mathrm{Ag}-\mathrm{Pd}$, $\mathrm{Pd}-\mathrm{Pd}$ bond lengths/ coordination numbers of the Pd, Au@Pd, and Au@Ag

$\mathrm{Au} / \mathrm{Ag}$ atoms. Moreover, the lower white line intensity of $\mathrm{Pd}$ atoms in $\mathrm{Au} @ \mathrm{Ag}_{\mathrm{ML}} @ \mathrm{Pd} \mathrm{NWs}$ reflects the higher d-band filling states compared to Pd in Au@Pd NWs, and confirms the gain of $d$-electrons from $\mathrm{Au} / \mathrm{Ag}$ atoms. Detailed first-shell fitting on the Pd k-edge extended X-ray adsorption fine structure (EXAFS) spectra revealed that all of the Pd atoms in Au@Pd and Au@ $\mathrm{Ag}_{\mathrm{ML}} @ \mathrm{Pd}$ NWs were primarily coordinated by 5 to $7 \mathrm{Au}$ or $\mathrm{Ag}$ atoms (Figure 3; Tables S1 and S2, Supporting Information). This value is much larger than the theoretical one ( 3 and 5 for $\mathrm{Pd} \mathrm{ML}$ on $\mathrm{Au}$ or $\mathrm{Au} / \mathrm{Ag}\{111\}$ and $\{110\}$ facet, respectively), revealing Pd atoms have relocated to the core-shell NWs, or diffused into the subsurface $\mathrm{Au}$ layer. ${ }^{[19]}$ The bond distance of $\mathrm{Au} /$ $\mathrm{Ag}-\mathrm{Pd}$ was $\approx 2.82 \AA$, which is in line with AuPd alloy NWs. ${ }^{[18 b]}$ However, compared with Pd NWs $(\approx 2.74 \AA)$, the Pd-Pd distance in the Au@Pd NWs is elongated to $\approx 2.84 \AA$. This is due to the surface strain of the Pd layer on the Au and Au@Ag NWs. Intriguingly, as the $\mathrm{Pd}$ atom coverage increased from $1 / 8$ to $1 / 2$ $\mathrm{ML}$, the coordination number $(\mathrm{CN})$ for $\mathrm{Pd}-\mathrm{Pd}$ also increased from 1.56 to 2.44, reflecting the growth of Pd ensemble. The existence of Pd ensemble on Au@Pd NWs, as well as their growth as Pd coverage increases, agrees with our SERS, and also expressed in the distinct hydrogen underpotential deposition $\left(\mathrm{H}_{\text {upd }}\right)$ and CO-stripping behaviors (Figures S14-S16, Supporting Information). ${ }^{\left[{ }^{5 b}\right]}$ By contrast, $\mathrm{Pd}-\mathrm{Pd}$ bond were only detected in Au@Ag $@$ ML $\mathrm{Pd}$ Nw when Pd coverage was 1/2 ML with a CN of 1.39 , the CN of Pd-Pd in the Au@Ag $\mathrm{Ag}_{\mathrm{ML}} @ \mathrm{Pd}$ NWs (1/8 ML) was less than 0.15 . The above EXAFS results decisively reveal that the Pd shell atoms primarily exist as isolated Pd atoms on the surface of the $\mathrm{Au} @ \mathrm{Ag}_{\mathrm{ML}} @ \mathrm{Pd}$ NWs. In addition, the $\mathrm{CN}_{\mathrm{Pd}-\mathrm{Pd}}$ value $(<3.0)$ was significantly lower than the $\mathrm{CN}_{\mathrm{Au} / \mathrm{Ag}-\mathrm{Pd}}$, suggesting that the majority of the $\mathrm{Pd}$ atoms were coated on the NWs as a monolayer (Figure S13, Supporting Information), and that Pd nanoparticles or segregated Pd atoms, e.g., 3D Pd ensembles, are absent. ${ }^{[18 c, 20]}$ This is further supported by the $\mathrm{Au}$ $\mathrm{L}_{\mathrm{III}}$-edge EXAFS spectra (Table S2, Supporting Information).
After consolidating the formation of isolated and continuous Pd atoms on Au@Ag and Au NWs, we further managed to roughly estimate their relative abundances based on spectroscopic differences (Table S3, Supporting Information see Discussion and Figure S17, Supporting Information). While similar quantitative measurements have been made using high resolution scanning tunneling microscopy(HR-STM) ${ }^{[5 a, b]}$ and HAADF-STEM, ${ }^{[4 b]}$ polycrystalline nanosurfaces, such as like NWs, are more technically complicated. For the Au@Pd NWs, the SERS signal from atop bound 2,6-DMPI only accounted for $8.21 \%, 10.84 \%, 12.29 \%, 8.27 \%$, and $1.05 \%$ of the total SERS signal for $1 / 16,1 / 8,1 / 4,1 / 2$, and $1.0 \mathrm{ML} \mathrm{Pd}$, respectively. However, these values were $16.03 \%, 25.18 \%, 46.33 \%, 59.42 \%$, and 44.92\% for their Au@ $\mathrm{Ag}_{\mathrm{ML}} @ \mathrm{Pd}$ analogs, respectively, which demonstrates that Pd primarily exists as isolated atoms on the $\mathrm{Au} @ \mathrm{Ag}_{\mathrm{ML}} @ \mathrm{Pd}$ NWs. It is deserved to note that the experimentally measured surface coverage of the $\mathrm{Au}$ and $\mathrm{Au} / \mathrm{Ag}$ atoms was lower than the theoretical value. The estimated Pd coverage of the Au@Pd NWs was also always higher than their Au@Ag $\mathrm{AL}_{\mathrm{M}} @ \mathrm{Pd}$ counterparts. One plausible explanation for this is that the Ag ML is effective in suppressing fusion of the Au surface. ${ }^{[5 c]}$ In addition, the covalent interaction between the NC group of 2,6-DMPI and the Pd atoms is stronger than with the $\mathrm{Ag}$ or $\mathrm{Au}$ atoms, which causes 2,6-DMPI to preferentially adsorb onto Pd. ${ }^{[17 b]}$

Once the atomic geometries of Pd atoms were quantitated, the role of $\mathrm{Pd}$ atomic geometry on the (electro) catalytic process was studied. The hydrogenation of 4-NP and the electrooxidation of ethanol were chosen as model reactions. In the reduction of $4-\mathrm{NP}$ by $\mathrm{KBH}_{4}$, Au, Au@Ag, and Pd NWs displayed limited activities with first-order rate constants of $1.5,4.3$, and $5.7 \times 10^{-3} \mathrm{~s}^{-1}$, respectively. After Pd was coated onto the Au surface, the reaction rate constants increased to 10.0 and $25.0 \times 10^{-3} \mathrm{~s}^{-1}$ for Au@Pd (1/16) and Au@Pd (1/8), respectively (Figure 4a; Figure S18, Supporting Information). 

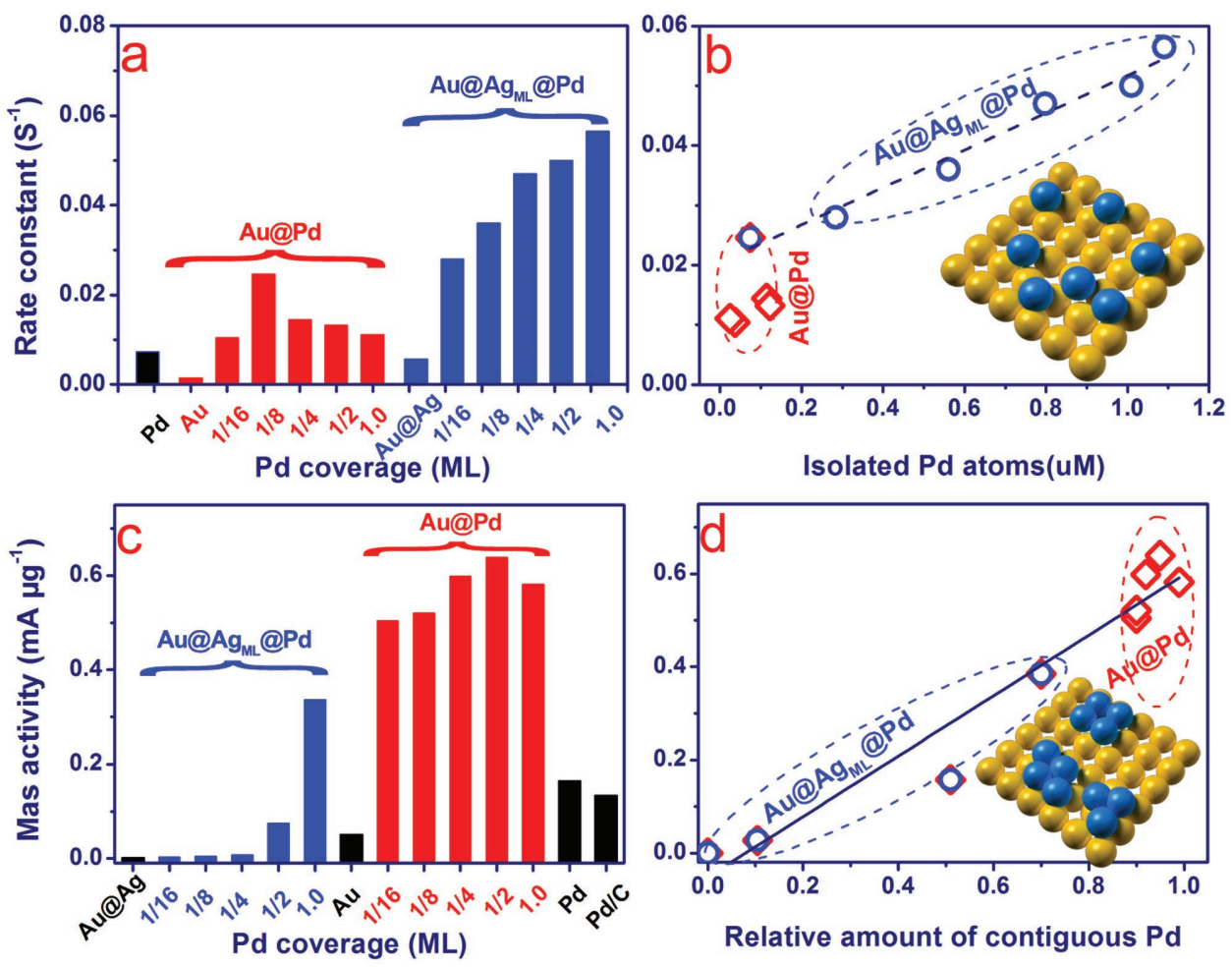

Figure 4. The catalytic performance of Au@Pd and Au@Ag $\mathrm{ML} @ P d$ NWs in the catalyzed reduction of a) 4-NP and c) the electrooxidation of 0.1 M ethanol. The observed activity in the reduction of 4-NP reduction is attributed to b) the isolated Pd atoms and d) the Pd ensembles in the case of ethanol electrooxidation.

However, further increasing the Pd coverage of the Au NWs reduced the rate constants (to $14.0,13.0$, and $11.0 \times 10^{-3} \mathrm{~s}^{-1}$ for 1/4, 1/2 and 1.0 ML Pd-coated Au@Pd NWs, respectively). Conversely, the rate constant in Au@Ag 9 ML $\mathrm{Pd}$ increased linearly from $28.0 \times 10^{-3} \mathrm{~s}^{-1}(1 / 16 \mathrm{ML})$ to $57.0 \times 10^{-3} \mathrm{~s}^{-1}$ (1.0 ML), which is one of the highest rate constants measured for this reaction (Table S4, Supporting Information). ${ }^{[21]}$ Notably, the deposition of Pd onto Au or Au@Ag surfaces may influence the intrinsic activities of the $\mathrm{Au}$ or $\mathrm{Au} / \mathrm{Ag}$ atoms themselves. This may account for the volcano-type dependence of the Au@ Pd NW catalytic activity on the amount of Pd coverage. However, this effect appears less significant in the case of the Au@ $\mathrm{Ag}_{\mathrm{ML}} @ P d \mathrm{NWs}$.

To further study how the atomic geometry of Pd influences catalytic activity, we evaluated the contributions of isolated atoms. Previous studies revealed that both $\mathrm{Pd} / \mathrm{Pt}$ nanoparticles and Pd clusters consisting of just a few Pd atoms are efficient catalysts for the reduction of 4-NP. ${ }^{[21]}$ However, the critical number of Pd atoms in the reactive center for this reaction has not yet been identified. The high activity of $\mathrm{Au} @ \mathrm{Ag}_{\mathrm{ML}} @ \mathrm{Pd}$ in this reaction, as well as the dominance of isolated $\mathrm{Pd}$ atoms in the catalyst indicates that a single Pd atom is sufficient for the transfer of surface hydrogen species to 4-NP. This hypothesis is supported by the linear relationship between the activity and the amount of isolated Pd atoms (Figure 4b, blue circles). Moreover, as shown in Table S4 (Supporting Information), the mass or surface area of the isolated Pd normalized activity is almost independent of Pd coverage, except in the case of Au@ Ag $g_{M L} @$ Pd with 1/16 ML Pd. In addition, the activity is much higher than those reported previously, ${ }^{[21]}$ and if the reaction conditions are taken into account (reductant concentration and temperature, see Supporting Information), our catalyst was at least 100-fold more active than that reported in the literature. For Au@Pd which has a limited number of isolated Pd atoms, a poor linear relationship between the activity and the amount of isolated Pd atoms was observed due the contribution of continuous Pd atoms (Figure 4b, red diamonds).

The differences in Pd atomic geometry also profoundly influence its electrocatalytic performance (Figure 4c; Figure S19, Supporting Information). Pd NWs and commercially available $\mathrm{Pd} / \mathrm{C}$, which is utilized as a benchmark catalyst, showed a mass activity of 0.16 and $0.13 \mathrm{~mA} \mathrm{\mu g}^{-1}$ surface Pd, respectively. After coating Pd on the Au NW surface as a submonolayer, the mass

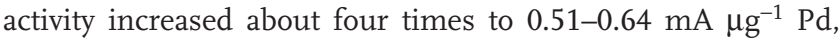
and almost irrelevant with $\mathrm{Pd}$ coverage. However, the mass activity of Pd in the Au@Ag $\mathrm{AL}_{\mathrm{ML}} @ \mathrm{Pd}$ NWs changed significantly with Pd coverage, which approached zero for the 1/16 ML to 1/4 ML Pd-coated Au@Ag NWs, and increased to 0.16 and $0.35 \mathrm{~mA} \mu \mathrm{g}^{-1} \mathrm{Pd}$ for the 1/2 ML and 1.0 ML Pd-coated Au@ Ag NWs, respectively. Considering the demonstrated evolution of the Pd atomic geometries with Pd coverage on the Au@Ag NWs, the change of the mass activity as a function of Pd coverage may be due to the fact that the Pd ensemble is the sole reactive center during ethanol electrooxidation. To further substantiate this conclusion, the mass activity of Pd in the Au@Pd and $\mathrm{Au} @ \mathrm{Ag}_{\mathrm{ML}} @ \mathrm{Pd} \mathrm{NWs}$ is correlated with the relative amount of continuous Pd atoms (Figure 4d). Again, the high $R^{2}$ value of 0.94 unambiguously suggests that the main active site is 
composed of continuous Pd atoms. Moreover, during the electrocatalysis process, the atomic geometry of $\mathrm{Pd}$ atoms is well preserved. This is revealed in the similar SERS spectra of 2,6DMPI adsorbed on as-synthesized Au@Pd and Au@Ag $@$ Pd NWs and NWs experienced 30 successive cyclic voltammograms (CVs) cycles (Figure S20, Supporting Information) and demonstrate the validation of our conclusion on the Pd geometry-dependent activity.

The main challenge in manipulating the atomic geometry of the shell metal atoms in a bimetal nanocatalyst is that the growth of metal ML generally begins from clusters or islands, and ultimately yields ensemble-dominated shell metal atoms. Meanwhile, albeit the central role of the atomic geometry of the active atoms in determining catalytic activity is well established, direct quantification of its relative contribution, and differentiating between the ligand, electronic and synergistic effects is difficult, in part due to underdeveloped methodologies for estimating the abundance of atoms within a given atomic geometry.

By measuring the SERS spectra of the molecular adsorbate, 2,6-DMPI, we demonstrated that feasibility of atomic-level manipulating Pd ML on ultrathin Au NW surfaces by the introducing an Ag ML between the Au core and the Pd shell. This atomic-scale structural modification was observed in the SERS (Figure 2) and EXAFS (Figure 3) spectra. As indicated by DFT calculations, ${ }^{[22]}$ the growth pattern of the MLs is governed by the binding energy of the deposited atoms. This binding energy is influenced by the facet energy, surfactants and coexisting ions, and a high binding energy favors the formation of isolated atoms. Therefore, the switch of the atomic geometry from Pd ensembles in Au@Pd to isolated atoms in Au@Ag $\mathrm{Ag}_{\mathrm{ML}} @ \mathrm{Pd}$ can be attributed to the stronger interaction between Pd atoms and $\mathrm{Au} @ \mathrm{Ag}_{\mathrm{ML}} \mathrm{NWs}$ over that of Pd atoms and $\mathrm{Au}$ NWs. Indeed, this was also reflected in the higher $d$-electron density of $\mathrm{Pd}$ atoms in Au@Ag $\mathrm{ML}_{\mathrm{L}} @ \mathrm{Pd}$ compared to Au@Pd NWs (Figure 2; Figure S14, Supporting Information), indicating that Au@Ag transfers more $d$-electrons to $\mathrm{Pd}$ atoms. ${ }^{[23]}$ Although $\mathrm{Au}$ atoms

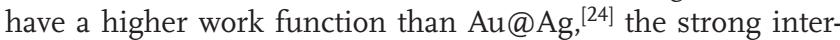
action between the $\mathrm{Au}$ atoms and the surfactant TX-114 (based on the more uniform and thinner diameter of Au NWs compared to Ag NWs stabilized by TX-114) ${ }^{[25]}$ may change or even reverse this order, thus further strengthening the Au@Ag-Pd interaction. As a consequence, the formation of isolated $\mathrm{Pd}$ atoms on the Au@Ag surface is more feasible than on the Au surface, where the formation of an extra Pd-Pd bond is necessary to stabilize the Pd atoms.

Additionally, measuring 2,6-DMPI chemisorption by SERS was the first demonstration of its unique ability to quantitatively estimate the relative abundance of catalytically active atoms within different atomic geometries. The proposed method is validated by the high linearity between the apparent activity (rate constant or mass activity) and the amount of reactive centers (Figure 4b,d), and the well-preserved Pd atomic geometry and its distribution during the catalysis process that revealed in the similar SERS spectra of 2,6-DMPI chemisorbed on fresh and used NWs catalyst (Figure S20, Supporting Information). Unlike previously reported microscope-based technologies, such as HRSTM and STEM, our method is applicable to polycrystalline structures ${ }^{[5 a, b]}$ and systems with a lower/similar
Z-contrast, ${ }^{[4 \mathrm{~b}]}$ as well as provide statistical macroscopic data, and potentially extends to analogous systems, especially noble metal@transit metal nanoarchitectures.

The provision of $\mathrm{Pd}$ atoms with different atomic geometries (Figures 2 and 3) and changed electronic (Figure S14, Supporting Information) and synergistic effects enable us to evaluate their relative contribution to the catalytic activity. Apparently, the high dependence/linearity between atomic geometry and the activity (Figure 4b,d) supports that the geometry effect (coordination number/bond length) takes priority over both the electronic and synergistic effects. This result appears more evident in the electrooxidation of ethanol. Indeed, the presence of $\mathrm{Au}$ or Ag atoms may influence the hybridization between the $\mathrm{Pd}$ atom and the $\mathrm{O}$ atoms in ethanol (electronic effect), facilitating the removal of CO-like intermediates, and providing reactive hydroxyl groups to remove the adsorbed acyl group from the Pd atoms (synergistic effect). ${ }^{[26]}$ This explains the much higher activity of Pd atoms on Au or Au@Ag surfaces compared to Pd NWs and Pd/C. However, two key steps in this process, the adsorption of ethanol and the removal of $\alpha-\mathrm{H}$, require at least two neighboring Pd atoms, ${ }^{[26 b]} \mathrm{ca}$., our DFT calculation revealed that high activation energy of $1.4 \mathrm{eV}$ is needed for the transform of ethanol into acetaldehyde (Figure S22, Supporting Information). Therefore, the atomic geometry of the Pd atoms takes a primary role in determining whether the $\mathrm{Pd}$ atoms are active during the electrooxidation of ethanol. This also means the introduced $\mathrm{Ag}_{\mathrm{ML}}$ between $\mathrm{Au}$ NWs core and $\mathrm{Pd}$ shell atoms only changes the atomic geometry of Pd atoms, but has less influence on their activity. In other word, Au and $\mathrm{Au} @ \mathrm{Ag}_{\mathrm{ML}}$ provides a similar coordination environment for Pd atoms, and thus similar electronic/synergic effect occurs on Pd atoms. This, in one hand, can be attributed to the similar physical/chemical property of $\mathrm{Au}$ and $\mathrm{Ag}$ atoms, and more importantly, Ag atoms present as monolayer on $\mathrm{Au} \mathrm{NWs,} \mathrm{and}$ $\mathrm{Au}$ plays a more decisive role in influence the catalytic activity of Pd atoms.

Meanwhile, electronic and synergy effects influence the activity of the reactive center as well, although not as decisive as the geometry. This provides a reasonable explanation for the superior activity of isolated Pd atoms in $\mathrm{Au} @ \mathrm{Ag}_{\mathrm{ML}} @ \mathrm{Pd}$ NWs over that of ensemble Pd in Au@Pd NWs (Figure 4a,b). Since the binding energy of 4-NP on reactive center depends on its composition (electronic effect), ${ }^{[27]}$ and noble metals, e.g., $\mathrm{Au}$ or $\mathrm{Ag}$, are capable of activating nitro group, while Pd atom facilitates the storage of hydrogen radical intermediates (synergy effect). ${ }^{[28]}$ Both of which indicates that the high activity observed for the isolated Pd atoms is a result of electronic and synergy effects. Compared to the Pd ensemble, isolated Pd atoms may be influenced strongly by $\mathrm{Au}$ or Ag atoms (Figure 2). This means that the observed geometry effect in the reduction of 4-NP may actually be due to an electronic or synergic effect. Notably, the Pd-Pd bond length is also an important influencer on the catalytic activity, ${ }^{[18 a]}$ however, due to insufficient data, its effect on the activity of Pd atoms cannot be elucidated at this time.

In summary, we have manipulated the atomic geometry of shell metal atoms by finely tuning the interactions between the shell and core atoms. As a result, the atomic geometry of shell Pd atoms was switched from ensemble dominated in Au@Pd 
to isolated atom dominated in Au@Ag 9 @ @Pd NWs. Based on these atomically designed $\mathrm{Pd}$ catalysts, the reactive center for the catalyzed reduction of 4-NP and electrooxidation of ethanol was identified experimentally. We anticipate this study will inspire further attempts to elucidate activity-structure relationships and design new generation of nanocatalysts from the atomic viewpoint.

\section{Experimental Section}

Synthesis of Ultrathin Au@Pd,Au@Ag, and Au@Ag ${ }_{M L} @ P d$ Core-Shell Nanowires: The ultrathin Au NWs were synthesized according to our reported procedure. ${ }^{[5]}$ For the preparation of Au@Pd core-shell NWs, $0.19,0.375,0.75,1.50$, or $3.0 \mathrm{~mL}$ of ice-cold $1.0 \times 10^{-3} \mathrm{M} \mathrm{Na}_{2} \mathrm{PdCl}_{4}$ solution was added into $10.0 \mathrm{~mL}$ dispersion of freshly synthesized $\mathrm{Au}$ NWs dropwisely while stirring. In each of these cases, the Pd coverage is $1 / 16,1 / 8,1 / 4 /, 1 / 2$ or $1.0 \mathrm{ML}$, respectively. The synthesis of Au@Ag NWs is similar to that of Au@Pd NWs, but replaces $\mathrm{Na}_{2} \mathrm{PdCl}_{4}$ with an equal amount of $\mathrm{AgNO}_{3}$. In the case of the Au@Ag $\mathrm{Ag}_{\mathrm{ML}} @ \mathrm{Pd} \mathrm{NWs}, 3.0 \mathrm{~mL}$ of $1.0 \times 10^{-3} \mathrm{M} \mathrm{AgNO}_{3}$ solution was added into a $10.0 \mathrm{~mL}$ dispersion of freshly synthesized $\mathrm{Au} \mathrm{NWs}$ while stirring, followed by the addition of $0.19,0.375,0.75,1.50$, or $3.0 \mathrm{~mL}$ of ice-cold $1.0 \times 10^{-3} \mathrm{M} \mathrm{Na}_{2} \mathrm{PdCl}_{4}$ solution.

Surface-Enhanced Raman Spectroscopy of 2,6-DMPI: To record the enhanced Raman spectra of 2,6-DMPI chemisorbed on the synthesized NWs, $100 \mu \mathrm{L}$ of $1 \times 10^{-3} \mathrm{~m} 2,6$-DMPI in ethanol was added into $5 \mathrm{~mL}$ NWs dispersions. The resulting solution was stirred for $5 \mathrm{~min}$ to allow for the formation of a 2,6-DMPI self-assembled monolayer on the NW surface. After removal the excess 2,6-DMPI by ultrafiltration and redispersed in $0.5 \mathrm{~mL}$ ethanol, $100 \mu \mathrm{L}$ of which was dipped onto a $\mathrm{Si}$ slide $\left(1 \mathrm{~cm}^{2}\right)$ to form a thin film. SERS experiments were conducted with a Renishaw InVia Raman microscope equipped with 532, 633, and $785 \mathrm{~nm}$ laser lines as excitation sources, and the Raman band of the silicon wafer at $520 \mathrm{~cm}^{-1}$ was used to calibrate the spectrometer.

XAS Study: The local coordination environment ( $\mathrm{CN}$ and bond length) of Pd atoms in Au@Pd and Au@AgML@Pd NWs was probed by XAS analysis. Besides the Pd-K edge spectra (Beamline 11ID-D of the Advanced Photon Source, Argonne National Laboratory), the Ag-k edge XAS spectra (Beamline 14W1 of the Shanghai Synchrotron Radiation Facilities, SSRF), as well as the Au $L_{11{ }^{-}}$edge XAS spectra (Beijing Synchrotron Radiation Facilities, BSRF) were also acquired to build a full model for Pd atoms. The XAS spectra were measured either in the fluorescence (Pd and $\mathrm{Ag}$ ) or transmission ( $\mathrm{Au}$ ) mode. Trace amount of sample was mixed with boron nitride (BN) and the mixture was grinded thoroughly before pressed into pellet. $\mathrm{Au}, \mathrm{Ag}$, and $\mathrm{Pd}$ foil were used for energy calibration. EXAFS data analysis was performed using the ATHENA and AETEMIS program in the Demeter computer package. ${ }^{[29]}$

Electrochemistry Experiments: The working electrodes were prepared by depositing $40 \mu \mathrm{L}$ of Au, or Au@Pd, Au@Ag, and Au@Ag 9 M @Pd NWs with the same amount of $A u$ (about $7.88 \mu \mathrm{g}$ ), or $4.24 \mu \mathrm{g} \mathrm{Pd} \mathrm{NWs} \mathrm{or} \mathrm{Pd/C}$ on the polished glassy carbon electrode $(5 \mathrm{~mm}$ i.d.). After drying in air, $3 \mu \mathrm{L}$ of $5 \%$ (w/v) Nafion solution (DE 530CS, DuPont) was dipped on the surface. The same suspension was used for Inductively Coupled Plasma Mass Spectrometry (ICP-MS) (Agilent 7500ce, USA) to determine the exact amount of $\mathrm{Au}$ and $\mathrm{Pd}$ loaded on the electrode.

The electrochemical test was carried out in a standard threeelectrode system with $\mathrm{Pt}$ wire and $\mathrm{Ag} / \mathrm{AgCl}$ as the counter electrode and reference electrode, respectively. Before recording any electrochemical data, the working electrode was activated with CVs from -1.0 to $0.45 \mathrm{~V}$ in $\mathrm{N}_{2}$-saturated $0.1 \mathrm{~m} \mathrm{KOH}$ until a steady $\mathrm{CV}$ was obtained. To evaluate the electrocatalytic activity of prepared Au@Pd and Au@Ag $g_{M L} @ P d$ $\mathrm{NWs}$, the electrooxidation of ethanol was performed in $0.1 \mathrm{M}$ ethanol + $0.1 \mathrm{M} \mathrm{KOH}$ at a scan rate of $50 \mathrm{mV} \mathrm{S}^{-1}$. The activities of the Pd NWs, $\mathrm{Au} / \mathrm{Pd} \mathrm{NW}$ s physical mixture, and Pd/C (Sigma Aldrich) were also tested.
Catalytic Hydrogenation of 4-Nitrophenol: In a typical experiment, the concentrations of 4-NP, $\mathrm{NaBH}_{4}$ (freshly prepared), and the nanocatalyst (total concentration of $\mathrm{Au}, \mathrm{Pd}$, and $\mathrm{Ag}$ ) were $0.1 \times 10^{-3}, 1.0 \times 10^{-3}$, and $0.02 \times 10^{-3} \mathrm{M}$, respectively. The ice-cold mixed solution was transferred into a quartz cuvette, and the absorbance at $400 \mathrm{~nm}$ was continuously measured for $300 \mathrm{~s}$. The first-order model was used to calculate the apparent rate constant

$k_{\text {app }}=-\operatorname{Ln}\left(A_{400(t)} / A_{400(t=0)}\right)$

\section{Supporting Information}

Supporting Information is available from the Wiley Online Library or from the author.

\section{Acknowledgements}

The authors acknowledge the anonymous reviewers for their constructive comments and suggestions. This work was financially supported by the National Key Research and Development Program of China (2016YFA0203102), Ministry of Science and Technology (2014CB932000), and the National Natural Science Foundation of China (21577157 and 91436102). The authors acknowledge Dr. Xiaoyi Zhang and Qingyu Kong (Argonne National Laboratory), Dr. Lirong Zheng (BSRF), and staff at the BL $14 \mathrm{~W} 1$ and BL14B1 beamline of the SSRF for their assistance during the XAS and the Syn-XRD measurements. The authors acknowledge Dr. Jitao Lv, Dr. Hui Zhang, Dr. Qian Liu, Prof. Sijin Liu, and Prof. Huiyu Liu for their helpful discussion.

Received: August 26, 2016

Revised: October 14, 2016

Published online: December 7, 2016

[1] M. Flytzani-Stephanopoulos, B. C. Gates, Annu. Rev. Chem. Biomol. 2012, 3, 545

[2] P. X. Liu, Y. Zhao, R. X. Qin, S. G. Mo, G. Y. Chen, L. Gu, D. M. Chevrier, P. Zhang, Q. Guo, D. D. Zang, B. H. Wu, G. Fu, N. F. Zheng, Science 2016, 352, 797.

[3] a) M. H. Shao, Q. W. Chang, J. P. Dodelet, R. Chenitz, Chem. Rev. 2016, 116, 3594; b) K. A. Kuttiyiel, K. Sasaki, Y. Choi, D. Su, P. Liu, R. R. Adzic, Energy Environ. Sci. 2012, 5, 5297; c) S. T. Hunt, M. Milina, A. C. Alba-Rubio, C. H. Hendon, J. A. Dumesic, Y. Román-Leshkov, Science 2016, 352, 974; d) S. Brimaud, R. J. Behm, J. Am. Chem. Soc. 2013, 135, 11716.

[4] a) X. G. Li, W. T. Bi, L. Zhang, S. Tao, W. S. Chu, Q. Zhang, Y. Luo, C. Z. Wu, Y. Xie, Adv. Mater. 2016, 28, 2427; b) B. T. Qiao, A. Q. Wang, X. F. Yang, L. F. Allard, Z. Jiang, Y. T. Cui, J. Y. Liu, J. Li, T. Zhang, Nat. Chem. 2011, 3, 634; c) X. F. Zhang, J. J. Guo, P. F. Guan, C. J. Liu, H. Huang, F. H. Xue, X. L. Dong, S. J. Pennycook, M. F. Chisholm, Nat. Commun. 2013, 4, 1924.

[5] a) M. S. Chen, D. Kumar, C. W. Yi, D. W. Goodman, Science 2005, 310, 291; b) F. Maroun, F. Ozanam, O. M. Magnussen, R. J. Behm, Science 2001, 293, 1811; c) R. Liu, J. F. Liu, Z. M. Zhang, L. Q. Zhang, J. F. Sun, M. T. Sun, G. B. Jiang, J. Phys. Chem. Lett. 2014, 5, 969 .

[6] a) K. Tedsree, T. Li, S. Jones, C. W. A. Chan, K. M. K. Yu, P. A. J. Bagot, E. A. Marquis, G. D. W. Smith, S. C. E. Tsang, Nat. Nanotechnol. 2011, 6, 302; b) Y. Lei, F. Mehmood, S. Lee, J. Greeley, B. Lee, S. Seifert, R. E. Winans, J. W. Elam, R. J. Meyer, P. C. Redfern, D. Teschner, R. Schlogl, M. J. Pellin, L. A. Curtiss, S. Vajda, Science 2010, 328, 224. 
[7] A. Corma, Angew. Chem., Int. Ed. 2016, 55, 6112.

[8] Y. T. Shi, C. Y. Zhao, H. S. Wei, J. H. Guo, S. X. Liang, A. Q. Wang, T. Zhang, J. Y. Liu, T. L. Ma, Adv. Mater. 2014, 26, 8147.

[9] a) J. K. Edwards, S. J. Freakley, A. F. Carley, C. J. Kiely, G. J. Hutchings, Acc. Chem. Res. 2014, 47, 845; b) H. Q. Liu, W. An, Y. Y. Li, A. I. Frenkel, K. Sasaki, C. Koenigsmann, D. Su, R. M. Anderson, R. M. Crooks, R. R. Adzic, P. Liu, S. S. Wong, J. Am. Chem. Soc. 2015, 137, 12597.

[10] K. Ding, A. Gulec, A. M. Johnson, N. M. Schweitzer, G. D. Stucky, L. D. Marks, P. C. Stair, Science 2015, 350, 189.

[11] a) R. N. Dhital, C. Karnonsatikul, E. Somsook, K. Bobuatong M. Ehara, S. Karanjit, H. Sakurai, J. Am. Chem. Soc. 2012, 134, 20250; b) F. Gao, D. W. Goodman, Chem. Soc. Rev. 2012, 41, 8009; c) B. P. Chaplin, M. Reinhard, W. F. Schneider, C. Schuth, J. R. Shapley, T. J. Strathmann, C. J. Werth, Environ. Sci. Technol. 2012, 46, 3655.

[12] A. E. Baber, H. L. Tierney, E. C. H. Sykes, ACS Nano 2010, 4, 1637.

[13] K. L. Jungjohann, S. Bliznakov, P. W. Sutter, E. A. Stach, E. A. Sutter, Nano Lett. 2013, 13, 2964.

[14] S. Alayoglu, A. U. Nilekar, M. Mavrikakis, B. Eichhorn, Nat. Mater. 2008, 7, 333.

[15] a) K. Sasaki, H. Naohara, Y. Choi, Y. Cai, W. F. Chen, P. Liu, R. R. Adzic, Nat. Commun. 2012, 3, 1115; b) D. L. Wang, H. L. Xin, Y. C. Yu, H. S. Wang, E. Rus, D. A. Muller, H. D. Abruna, J. Am. Chem. Soc. 2010, 132, 17664; c) J. F. Huang, Y. H. Zhu, M. Lin, Q. X. Wang, L. Zhao, Y. Yang, K. X. Yao, Y. Han, J. Am. Chem. Soc. 2013, 135, 8552.

[16] a) Y. Ding, F. R. Fan, Z. Q. Tian, Z. L. Wang, J. Am. Chem. Soc. 2010, 132, 12480; b) S. Khanal, G. Casillas, J. J. Velazquez-Salazar, A. Ponce, M. Jose-Yacaman, J. Phys. Chem. C 2012, 116, 23596; c) H. Huang, L. Zhang, Z. H. Lv, R. Long, C. Zhang, Y. Lin, K. C. Wei, C. M. Wang, L. Chen, Z. Y. Li, Q. Zhang, Y. Luo, Y. J. Xiong, J. Am. Chem. Soc. 2016, 138, 6822 .

[17] a) S. M. Gruenbaum, M. H. Henney, S. Kumar, S. Z. Zou, J. Phys. Chem. B 2006, 110, 4782; b) J. Hu, M. Tanabe, J. Sato, K. Uosaki, K. Ikeda, J. Am. Chem. Soc. 2014, 136, 10299.
[18] a) X. M. Wang, Y. Orikasa, Y. Takesue, H. Inoue, M. Nakamura, T. Minato, N. Hoshi, Y. Uchimoto, J. Am. Chem. Soc. 2013, 135, 5938; b) X. W. Teng, Q. Wang, P. Liu, W. Han, A. Frenkel, W. Wen, N. Marinkovic, J. C. Hanson, J. A. Rodriguez, J. Am. Chem. Soc. 2008, 130, 1093; c) S. W. T. Price, J. M. Rhodes, L. Calvillo, A. E. Russell, J. Phys. Chem. C 2013, 117, 24858.

[19] J. D. Padmos, M. L. Personick, Q. Tang, P. N. Duchesne, D. E. Jiang, C. A. Mirkin, P. Zhang, Nat. Commun. 2015, 6, 7664.

[20] V. V. Srabionyan, A. L. Bugaev, V. V. Pryadchenko, L. A. Avakyan, J. A. vanBokhoven, L. A. Bugaev, J. Phys. Chem. Solids 2014, 75, 470 .

[21] a) J. L. Zhang, X. Wang, Y. Fu, Y. Han, J. Y. Cheng, Y. Q. Zhang, W. Li, Langmuir 2013, 29, 14345; b) M. Schrinner, M. Ballauff, Y. Talmon, Y. Kauffmann, J. Thun, M. Moller, J. Breu, Science 2009, $323,617$.

[22] a) E. V. Carino, H. Y. Kim, G. Henkelman, R. M. Crooks, J. Am. Chem. Soc. 2012, 134, 4153; b) Y. Zhang, Y. C. Hsieh, V. Volkov, D. Su, W. An, R. Si, Y. M. Zhu, P. Liu, J. X. Wang, R. R. Adzic, ACS Catal. 2014, 4, 738.

[23] J. S. Jirkovsky, I. Panas, E. Ahlberg, M. Halasa, S. Romani, D. J. Schiffrin, J. Am. Chem. Soc. 2011, 133, 19432.

[24] F. R. Fan, D. Y. Liu, Y. F. Wu, S. Duan, Z. X. Xie, Z. Y. Jiang, Z. Q. Tian, J. Am. Chem. Soc. 2008, 130, 6949.

[25] R. Liu, J. F. Sun, D. Cao, L. Q. Zhang, J. F. Liu, G. B. Jiang, Chem Commun. 2015, 51, 1309.

[26] a) L. Y. Chen, N. Chen, Y. Hou, Z. C. Wang, S. H. Lv, T. Fujita, J. H. Jiang, A. Hirata, M. W. Chen, ACS Catal. 2013, 3, 1220; b) G. F. Cui, S. Q. Song, P. K. Shen, A. Kowal, C. Bianchini, J. Phys. Chem. C 2009, 113, 15639.

[27] Z. D. Pozun, S. E. Rodenbusch, E. Keller, K. Tran, W. J. Tang K. J. Stevenson, G. Henkelman, J. Phys. Chem. C 2013, 117, 7598.

[28] J. F. Huang, S. Vongehr, S. C. Tang, X. K. Meng, J. Phys. Chem. C 2010, 114, 15005 .

[29] a) M. Newville, J. Synchrotron Radiat. 2001, 8, 322; b) B. Ravel, M. Newville, J. Synchrotron Radiat. 2005, 12, 537. 